\title{
Laboratory study of electromagnetic initiation of slip
}

\author{
Tamaz Chelidze, Nodar Varamashvili, Marina Devidze, Zurab Tchelidze, Victor Chikhladze \\ and Temur Matcharashvili \\ Institute of Geophysics, Georgian Academy of Sciences, Tbilisi, Georgia
}

\begin{abstract}
Recently Russian seismologists reported the triggering effect of MHD soundings on microseismic activity in the Central Asia test area. The paper focuses on an experimental test of the possibility of triggering the mechanical instability of a system that is close to critical state by a series of electromagnetic pulses. The mechanical system consisted of two pieces of rock; the upper piece can slip on the fixed supporting sample if the latter one is tilted up to the critical angle. In this state, the triggering of mechanical instability by some weak impact such as electrical pulse became more probable. The slope of support in the experiment is an analogue of tectonic stress in natural conditions. The preliminary experiments, carried out in a dry environment, at the humidity of atmosphere $30-50 \%$, show that a strong EM-pulse induces sliding of a sample of rock (granite, basalt, labradorite) placed on the supporting sample which is inclined at the slope close to, but less than, the critical angle with a probability 0.07 .
\end{abstract}

Key words electromagnetic triggering - mechanical instability

\section{Introduction}

In the experiments, initially aimed at finding resisitivity precursors of strong earthquakes in the upper layers of Earth crust by MHDsounding, an unexpected effect of microseismicity activation was discovered (Tarasov et al., 1999). The field of MHD-dipole is quite small at distances in the order of $100 \mathrm{~km}$, where the effect was observed: for resistivity of rocks of the order of $100-1000 \Omega \cdot \mathrm{m}$ (which is the resisitivity of rocks at the depth $5-10 \mathrm{~km}$ in the test area, according to Tarasov et al. (1999), at the distance of the order of $100 \mathrm{~km}$ from the dipole source (this is the distance to the area where the activation of microseismicity was

Mailing address: Dr. Tamaz Chelidze, Institute of Geophysics, Georgian Academy of Sciences, 1 Alexidze str., Tbilisi 380093, Georgia; e-mail: chelidze@ig.acnet.ge noted) the intensity of dipole field for the experimental source is of the order of $10^{-5}-10^{-6}$ $\mathrm{V} / \mathrm{m}$. The second peculiarity of the activation effect was that it occurred with 2-4 day delay after EM impact. The laboratory data on acoustic emission during compression of artificial samples (Sobolev et al., 2000) confirm the possibility of initiation of fractures by strong electromagnetic (EM) field. Thus it is necessary to find at least a phenomenological physical model explaining these two important details: long-range action and delayed response.

\section{Phenomenological approach}

The system, which is close enough to the critical state, can manifest anomalous sensitivity to small external impacts. According to recent investigations, the Earth's crust in seismically active regions can be in a critical state or in a state of self-organized criticality (Bak et al., 1988; Scholtz, 1990). This can explain the known phenomenon of seismic activation on filling large reservoirs; this additional loading gives an 
insignificant contribution to the existing tectonic strains. Other examples are seismicity activation by pumping of water in the boreholes (Sibson, 1994) and remote aftershocks of Landers earthquake. According to King et al. (1994), aftershocks recorded very far from the epicenter of the earthquake were generated by only one-half bar increment in stress provided by the mainshock.

One of the possible mechanisms can be the direct dielectric breakdown of rocks driven by tectonic stress to the critical state. That means that the EM pulse should be strong enough, which can be expected only in the near-source zone. In the far zone, the pulse should be amplified in order to cause dielectric breakdown. Dielectric breakdown as a rule is accompanied by emission of elastic waves. This class of models, which can be called «underground thunderstorms», was advanced by A. Vorobyev et al. (1975). The EM field amplification can be realized in following ways:

1) Amplification of EM field by wedge-type inclusions - Local electric field near inclusion with conductivity $g_{2}$, much larger than conductivity of the host medium $g_{1}$, can exceed the intensity of the mean macroscopic field. The largest effect is expected for the needle- or lensshaped inclusions. It is well known that elongated conductive impurities in transformer oil enhance dielectric breakdown (Kharitonov, 1983). As the cracks in rocks are usually saturated with conducting pore fluid and have the form of lens or needle, some amplification of the applied field can be expected: theoretically at the tip of the needle, the local field intensity is infinitely high.

In order to assess the effect more precisely, some experiments were performed on a physical (two dimensional) model. The aluminum «double wedge» inclusion was glued on the bottom of a flat glass vessel, which was filled with tap water. Specific resistivities of metal and fluid were $2.810^{-8} \Omega \cdot \mathrm{m}$ and $100 \Omega \cdot \mathrm{m}$ respectively, i.e. the resistivity ratio was $10^{10}$. The voltage $20 \mathrm{~V}$ was applied to electrodes, separated by $38 \mathrm{~cm}$; the uniform potential gradient is thus $0.526 \mathrm{~V} / \mathrm{cm}$.

Figure 1 shows electric field intensity $E$ along profiles, parallel to the long axis, which are far from the inclusion (1-1), close to it (2-2) and crossing it (3-3).

We see that at the tip of the inclusion, the local field can be orders of magnitude larger than the mean macroscopic field; that can give amplification of intensity from $\mu \mathrm{V}$ to $\mathrm{mV}$ which still is not enough to cause dielectric breakdown: the intensity should be of the order of $10^{4}-10^{5}$ $\mathrm{V} / \mathrm{cm}$.

2) Amplification of EM field in the random lattice model - Besides above discussed «wedge» model, there is another class of models that also explain local amplification of applied voltage, namely, percolation (random lattice) models. It has been shown (De Archangelis et al., 1986; Benguigi, 1988) that distribution of voltages on the random lattice of resistors and insulators is multifractal and at the percolation threshold $p_{c}$, the maximal voltage drop $V_{\max }$ occurs on singly connected (so called red) bonds, which carry the total current passing through the network. There are two models of electrical breakdown of the random lattice, dielectric and fuse. In the first model, the system is a conductor-loaded insulator. An insulating element becomes conducting (breaks) at a voltage higher than $V_{t h}$. Then, the whole system becomes conducting at the breakdown voltage $V_{b}$, which strongly depends on the concentration of conducting bonds $p$. In the second (fuse) model, the system is an insulator-loaded conductor. Here the conducting element is fused, i.e. it becomes an insulator if the current flowing through it is larger than the threshold current $J_{t h}$. Again, the whole system fuses (becomes an insulator) on carrying the current $J_{b}$ which strongly depends on the concentration of conductors $p$.

Near percolation threshold $p_{c}$ when the infinite cluster of conducting bonds spans the whole sys-tem, the breakdown voltage $V_{b}$ has a typical power law form (Archangelis et al, 1986; Benguigi, 1988)

$$
V_{b}=\left(p-p_{c}\right)^{v}
$$

and goes to zero as $p \rightarrow p_{c}$. Here $v$ is the exponent of correlation length $\xi$. For two-dimensional 


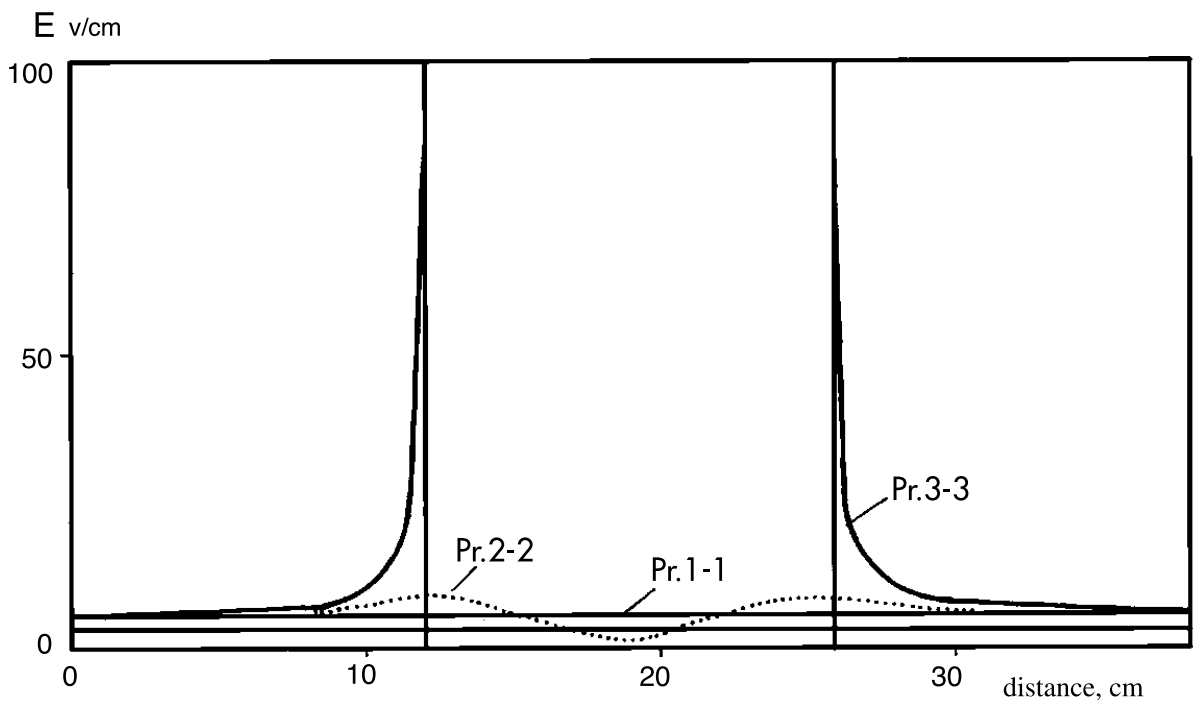

Fig. 1. Electric field intensity (E) due to elongated (lense-like) conductive inclusion along profiles 1-1, 2-2 and 3-3 (see text).

systems $v=4 / 3$. According to (2.1) at $p \rightarrow p_{c}$ the breakdown (applied) voltage becomes very low.

The geological formations can be considered as mosaics of insulating (minerals) and conducting (brine-saturated pores and cracks) components, which in some areas are close to the percolation threshold. This in principle can explain the local breakdown phenomenon, as at $p \rightarrow p_{c}$ the intensity of the field, necessary for initiation of breakdown tends to zero. Thus, theoretically the local breakdown voltage can be achieved at very small applied field if the system is close to the percolation threshold.

The EM-activation mechanism can also be related to pore fluid action.

3) Seismohydraulic mechanism of activation of seismicity - We have noted that in order to initiate fracturing by EM pulses, the system should be close to the critical state; in our case close to the state of mechanical instability. The critical shear stress on the fault $\tau_{c}$ is

$$
\tau_{c}=c+\mu\left(\sigma_{n}-P_{f}\right)
$$

where $c$ is resistance to fault displacement due to its partial cementation, $\mu$ is friction coefficient, $\sigma_{n}$ is the stress component normal to the fault plane, $P_{f}$ is fluid pore pressure. It is evident that, in order to provoke mechanical instability, EMpulse should affect at least one of parameters of the above formula and so change $\tau_{c}$. It seems that $\mu, \sigma_{n}$ and $P_{f}$ can be affected by EM pulse. For example, due to the piezoelectric effect $\mu$ and $\sigma_{n}$ can be favorably changed. The weakening of faults under EM impact can also be connected with interaction of pore fluid and mineral backbone, namely:

a) Application of EM pulse can drive pore fluid into the «dry» cracks. This decreases surface fracture energy, which means enhancement of crack growth either by Griffith's model of fracture or just by decrement of friction coefficient $\mu$.

b) Electrical impact can provoke electrokinetic flow in the porous rock (electroosmosis) and thus increase pore pressure $P_{f}$.

c) Strong EM impact can generate so called electrohydrodynamic effect (EHD) which was discovered in the 1950's (Nesvetailov and Serebryakov, 1966). EHD effect means that water saturated porous solid can be destroyed by strong 
enough EM pulses; this has even found industrial applications. The mechanism of EHD effect is not exactly known. The most popular explanation is cavitation or generation of small gas bubbles by applied EM pulse; their collapse generates transient stress fields in the fluid, which destroys a solid.

The first two mechanisms in principle can explain the considerable time lag between impact and (remote) response as the pore fluid migration or diffusion under electric field is a relatively slow process.

Rigorous quantitative testing of the above hypotheses is very difficult as all these models contain some parameters that cannot be measured exactly: say, closeness to the percolation thresh- old of geological formation at the depth $5-10 \mathrm{~km}$, water content, stress state, etc.

Thus the most important thing now is to prove the potential of EM pulses to trigger mechanical instability in laboratory experiments and to create reasonable physical models of the phenomenon. The present paper is devoted to experiments on EM initiation of slip in relatively dry environment, at the humidity of atmosphere $40-50 \%$.

\section{Experimental set up}

The experimental set up was designed in such a manner that the mechanical system could easily be driven to the critical state where the triggering

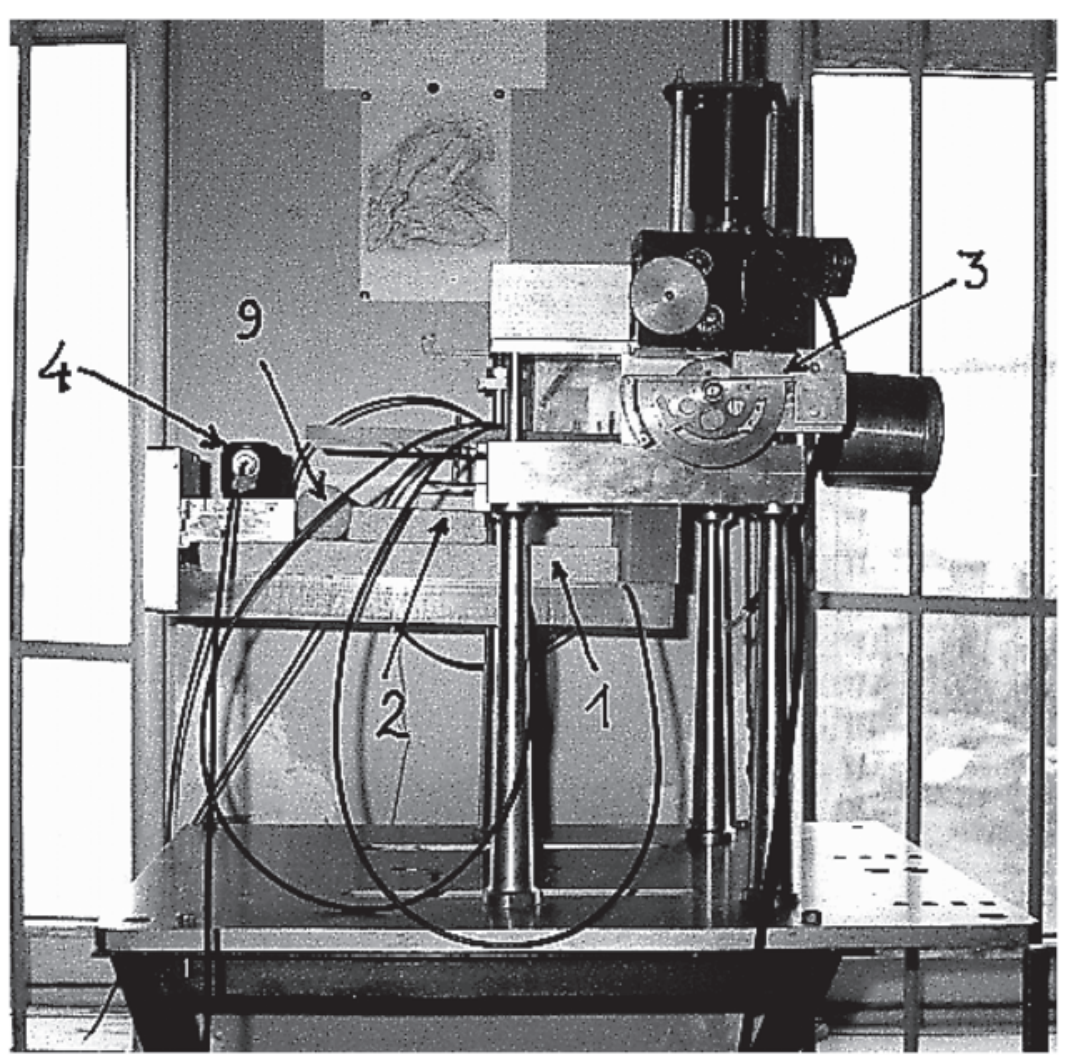

Fig. 2. The mechanical part of the experimental set up: $1=$ supporting sample; $2=$ sliding sample; $3=$ slope regulating unit; $4=$ acoustic sensors; $9=$ sponge for shock . 
of mechanical instability by some weak impact such as electrical pulse became more probable. The system consists of two pieces of rock; the upper piece can slip on the fixed supporting sample if the latter one is tilted up to the critical angle. The slope of support in the experiment is an analogue of tectonic stress in natural conditions.

The set up can be used for experiments in various conditions, «dry» and «wet». In the latter case the sample holder should be immersed in the bath with water. fig. 2 .

The general view of the set up is shown in

\subsection{Mechanical part}

The device brings the support-slider system to the critical state, that is, to the critical slope of the support. The regulation and fixing of the tilt is realized by means of the vernier system with an accuracy of $15^{\prime}$. The second important detail is the acoustic emission sensor, which responds to elementary slip events of the upper sample on the support. It is the relatively low frequency sensor $(25-100 \mathrm{kHz})$. The sensor was glued to the supporting (fixed) sample.

\subsection{Electrical part}

The electrical part consists of a EM pulse generator and acoustic signal amplifier. For experiments, it was necessary to assemble a system generating high intensity $\mathrm{AC}$ and $\mathrm{DC}$ fields. For this, a special high voltage linear amplifier was built. It has two outputs, for AC and DC voltages. The signal from the standard generator with amplitude $0.5-5 \mathrm{~V}$ is applied to the input of the amplifier and goes out from the output with amplitude up to $1300 \mathrm{~V}$. The high voltage pulses are applied to co-planar electrodes, placed under the supporting rock sample. The electrodes are of various forms (fig. 3) and can be connected in various combinations.

Another amplifier was designed to record acoustic signals from the sensors, which respond to the slip events. This is a two-channel low-noise amplifier; the amplification rate of channels can

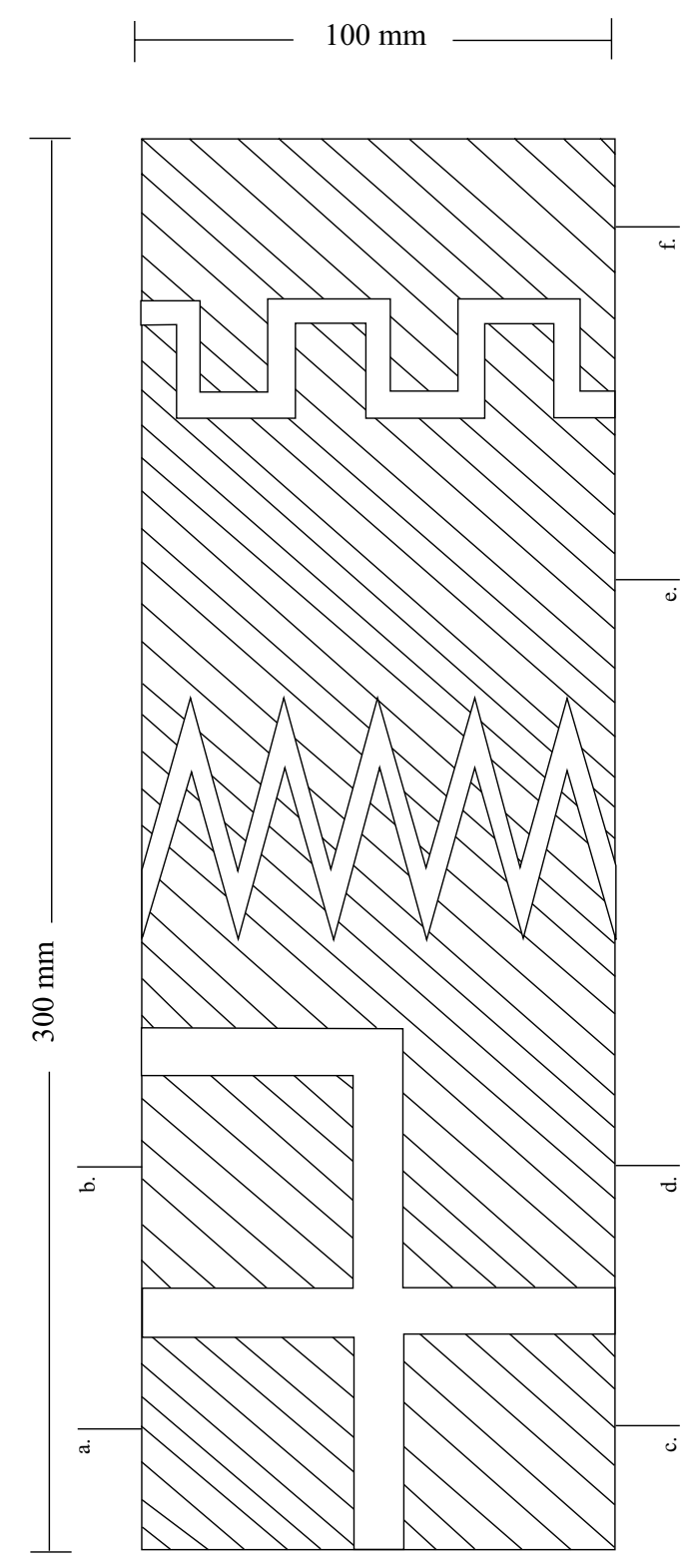

Fig. 3. The configuration of electrodes, located under the supporting sample.

be regulated separately. The amplifier's output voltage is sufficient for recording acoustic signals by a sound card of the PC. In the scheme, the output clipper is installed, which protects PC 
input from voltage overload and indicates overload of low-noise amplifier as well. The PC used was a Pentium II. The scanning of the process was performed on the frequency $96 \mathrm{kHz}$, i.e. the sampling rate was $1 / 96000 \mathrm{~s}$.

\subsection{Samples}

The supporting and the slipping blocks were prepared from glass, basalt, labradorite and granite. In all experiments, the support and the sliding block were of the same material. Their finish after sewing was either crude (basalt) or fine (other samples). The height of surface protuberances in the former case was in the range 0.1-0.2 $\mathrm{mm}$ and in the latter case - 0.05$0.1 \mathrm{~mm}$.

\section{Experimental results}

\subsection{The critical angle measurements}

The natural critical slopes of slip for various couples of rocks were determined. For each sample, 20 measurements of critical angle were made. The results are shown in the fig. 4 . For all samples, except basalt, the critical angle was in the range $8-13^{\circ}$; for basalt it was $25^{\circ}$. The data are scattered around these mean values with a standard deviation of the order of the $0.5^{\circ}$. The experiment on glass discs shows that the area and thickness of samples do not change the critical angle notably.

\subsection{Assessment of friction coefficient}

From elementary physics it is known that the friction coefficient $\mu$ for the body, placed on the inclined plane, can be calculated from the condition that at the critical angle $\alpha_{c}$, when the body slips down the plane, the friction force $F$ equals zero

$$
F=m g\left(\mu \cos \alpha_{c}-\sin \alpha_{c}\right)=0
$$

$\alpha$ where $m$ is the mass of the upper (slipping) sample, $g$ is gravity force acceleration, or

$$
\mu=\operatorname{tg} \alpha_{c}+0(\alpha)
$$

Using data on the critical angle, obtained on the tested materials (fig. 4) the average values of friction coefficient for finished samples are between 0.14 and 0.23 and for the basalt sample $\mu=0.47$.

\subsection{Laboratory modelling of EM-quakes (experimental procedure and case studies)}

The main objective of experiments was to find out whether the EM-pulse could indeed displace the rock sample placed on the supporting sample at the slope of support less than but close to the critical slip angle.

Surprisingly, all tested samples except glass were eventually displaced (slipped) by strong EM-pulses. The amplitude of DC-pulses was $1200 \mathrm{~V}$; duration from 5 to $10 \mathrm{~s}$, and interval between pulses was also from 5 to $10 \mathrm{~s}$. In the following experiments, the negative pole of the generator was applied to electrodes $a$ and $b$, and the positive one to electrodes $c$ and $d$ (fig. 3).

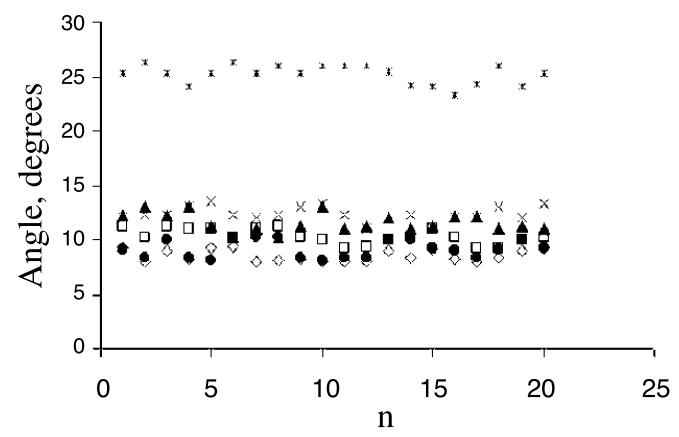

Fig. 4. The values of critical angles of slip for the following pairs of materials: Stars = basalt; diamonds $=$ glass (diameter $2 \mathrm{~cm}$, thickness $10 \mathrm{~mm}$ ); quadrangles = glass (diameter $7.5 \mathrm{~cm}$, thickness $10 \mathrm{~mm}$ ); triangles = glass (diameter $9.5 \mathrm{~cm}$, thickness $15 \mathrm{~mm}$ ); crosses = glass $($ diameter $14 \mathrm{~cm}$, thickness $12 \mathrm{~mm}$ ); circles = labradorite. 
After finding the critical angle, the slope of support was decreased by $0.1-2^{\circ}$. In this state, the upper sample was not moving for many hours (days), which means that other sources of instability such as building vibration by trucks, elevator, wind, etc. are not strong enough to initiate the slip. As the critical angle for the rough surface varyies (it is impossible to exactly reproduce the arrangement of asperities between support and slipping block), the sample was kept for at the angle $\alpha<\alpha_{c}$ for $10 \mathrm{~min}$ and only after this subjected to EM-impact. That allows correct assessment of the statistics of EM-activation, as the probability of slip in time intervals without EM-impact can be compared with that in time intervals covering the whole EM-activation period (including gaps between pulses). Actually, the probability of slip without EM-impact at $\alpha<\alpha_{c}$ was zero: no slip was observed during 10 min rest periods.

Application of EM-pulse initiates slip either during pulses (i.e. in the active phase), or after them (i.e. in the passive phase). On the experimental recordings (figs. 5 to $7 \mathrm{a}, \mathrm{b}$ ), the horizontal axis is the time and $y$-axis is the amplitude of $\mathrm{AE}$ (upper trace) or applied EM field pulse duration (lower trace). When the EM field is switched on, the trace is bold, during gaps it is thin. The vertical bars on AE channel correspond to switching on and off of the EM field; they are caused by the sound of the switch. On both channels, the $y$-axis shows the amplitude in decibels $(\mathrm{dB})$. A zero value of $\mathrm{dB}$ scale corresponds to maximum absolute amplitude and negative

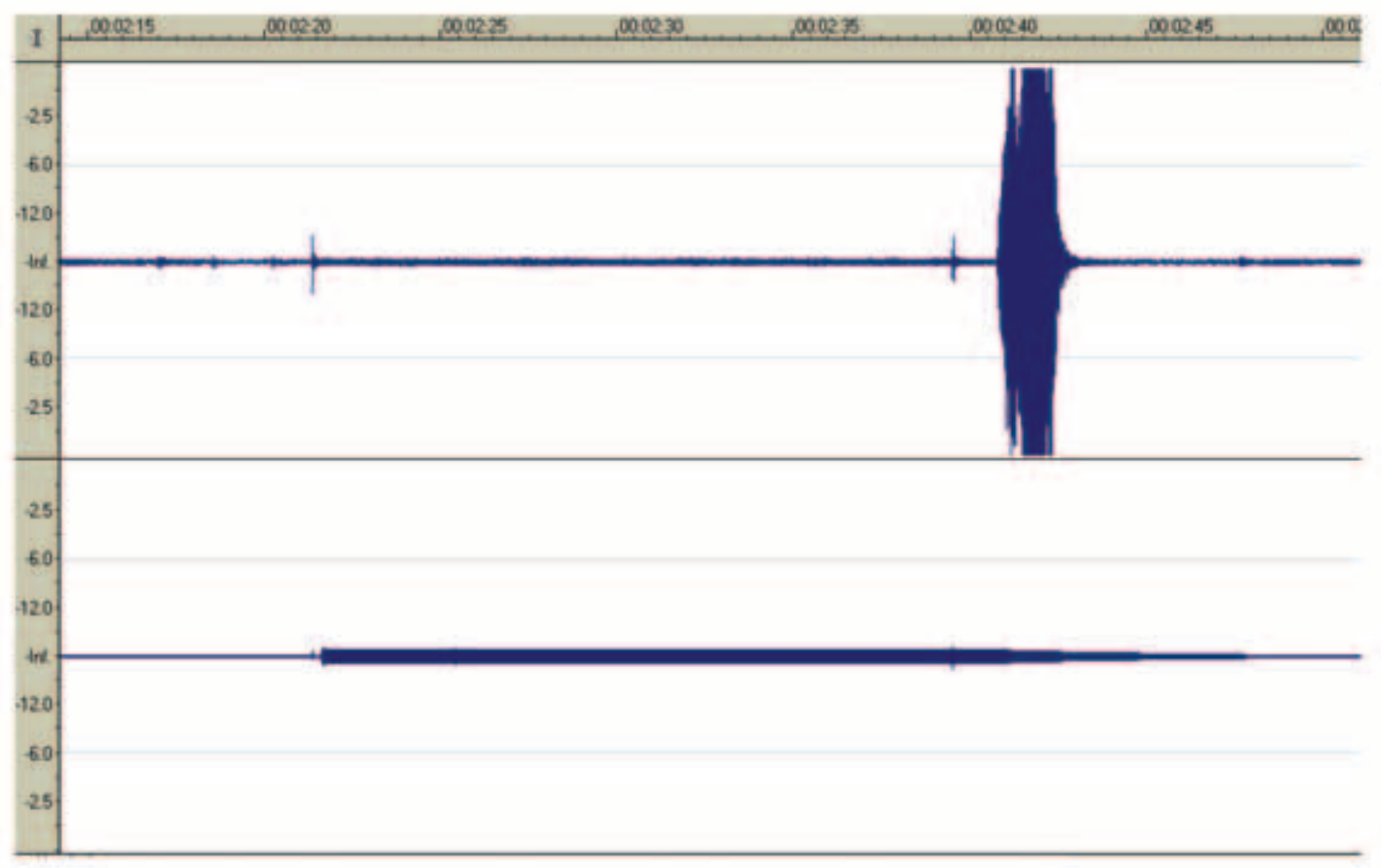

Fig. 5. EM-slip on the basalt sample. Slip happens in the passive phase. In figs. 5, 6 and $7 \mathrm{a}, \mathrm{b}$ on both channels the $y$-axis shows the amplitude in decibels $(\mathrm{dB})$. A zero value of $\mathrm{dB}$ scale corresponds to maximum absolute amplitude and negative infinity ( - Inf) corresponds to the complete absence of a signal. On the time axis of figs. 5, 6 and $7 \mathrm{a}, \mathrm{b}$ the first two numbers correspond to hours, next two positions show minutes and the last numbers are for seconds and milliseconds from the beginning of the experiment (for example, 00.02.40.800 means $00 \mathrm{~h}, 02 \mathrm{~min}$, $40 \mathrm{~s}$ and $800 \mathrm{~ms}$ ). 
infinity (-Inf) corresponds to the complete absence of a signal. As the figures are screen print outs, time marks are not very clear; corresponding comments are given in captions. Small perturbations of $\mathrm{AE}$ channel during gaps are caused by action of PC and generator fans.

The case histories of slip events are as follows (here only some typical cases are shown):

1) Figure 5 presents the history of the test on the samples of basalt. In this test, the AE sensor was applied to the lower side of the organic glass frame under the supporting sample. Here and in the following tests, the upper trace shows the acoustic sensor's output and the lower one records the switching on and off of the EM-pulse (the thick part of the lower trace corresponds to the active phase, i.e. current on). In this case, slip was initiated in the passive phase.

2) Figure 6 shows in more detail the initial stage of slip on the same basalt sample.

3) Figure 7a,b presents the results of labradorite sample testing. The EM-induced slip has been observed soon after the application of pulse during the active phase.

\subsection{Assessment of Electrical Field Intensity (EFI) on the slip surface}

In the following experiments, only basalt samples were studied.

The voltage applied to the samples was $1200 \mathrm{~V}$; nevertheless it is important to know the electrical field, acting on the slip surface.

The calculation of electrical field on the slip surface can be carried out using standard equations of electrostatics. For the pair of co-planar electrode systems used in our experiment, the EFI on the height $2.5 \mathrm{~cm}$ above the electrodes, put under voltage $1250 \mathrm{~V}$, varies from approximately $10 \mathrm{~V} / \mathrm{cm}$ to $40 \mathrm{~V} / \mathrm{cm}$ if the space is filled by material with dielectric constant 5 (characteristic for basalt in room conditions).

The calculated values are for homogeneous media. Real samples are very heterogeneous; that means that the EFI should also be measured experimentally. EFI on the edges of interface between supporting and sliding samples was measured by a digital multimeter with accuracy $\pm 0.01 \mathrm{~V}$. The voltage was measured relative to

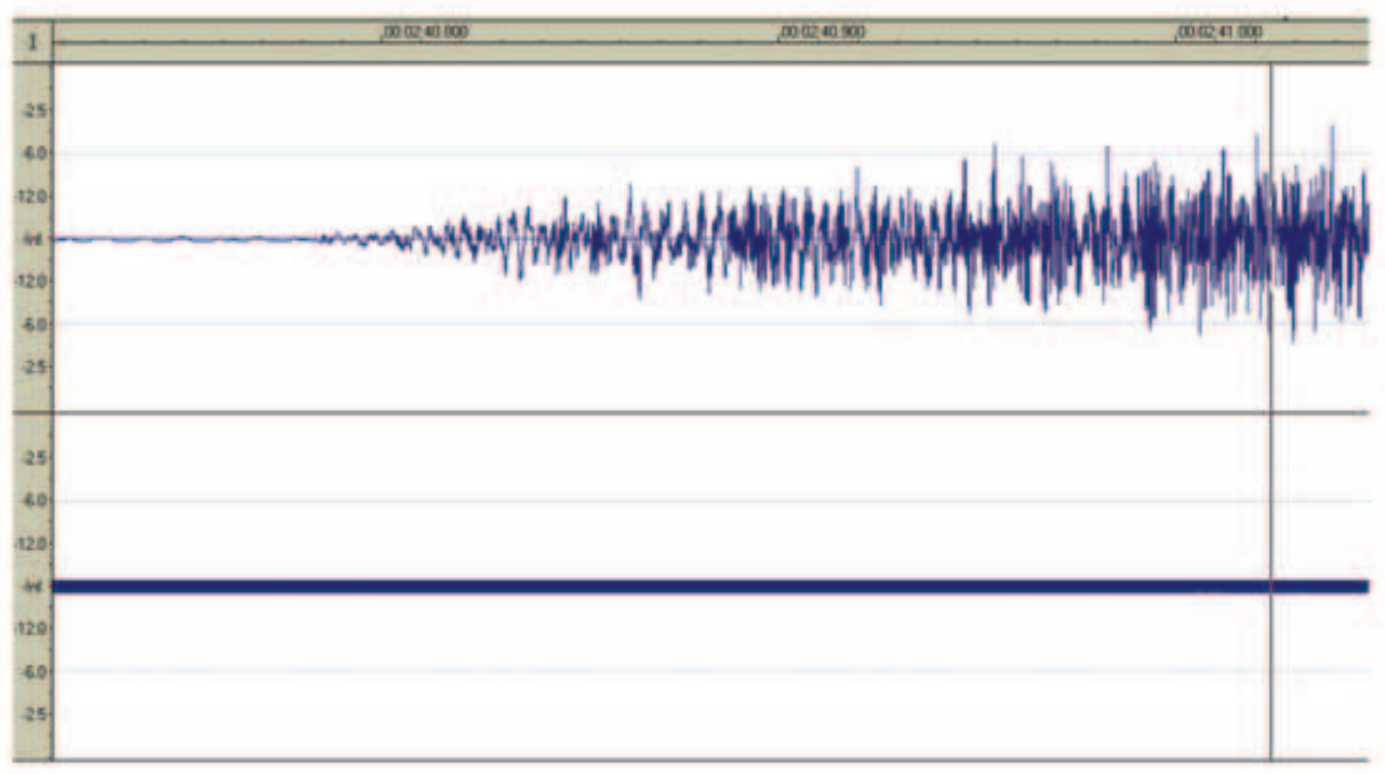

Fig. 6. EM-slip on the same basalt sample test; the time scale of the initial stage of slip (from 00.02.40.800 to 00.02.4.000) is stretched. 
(a)

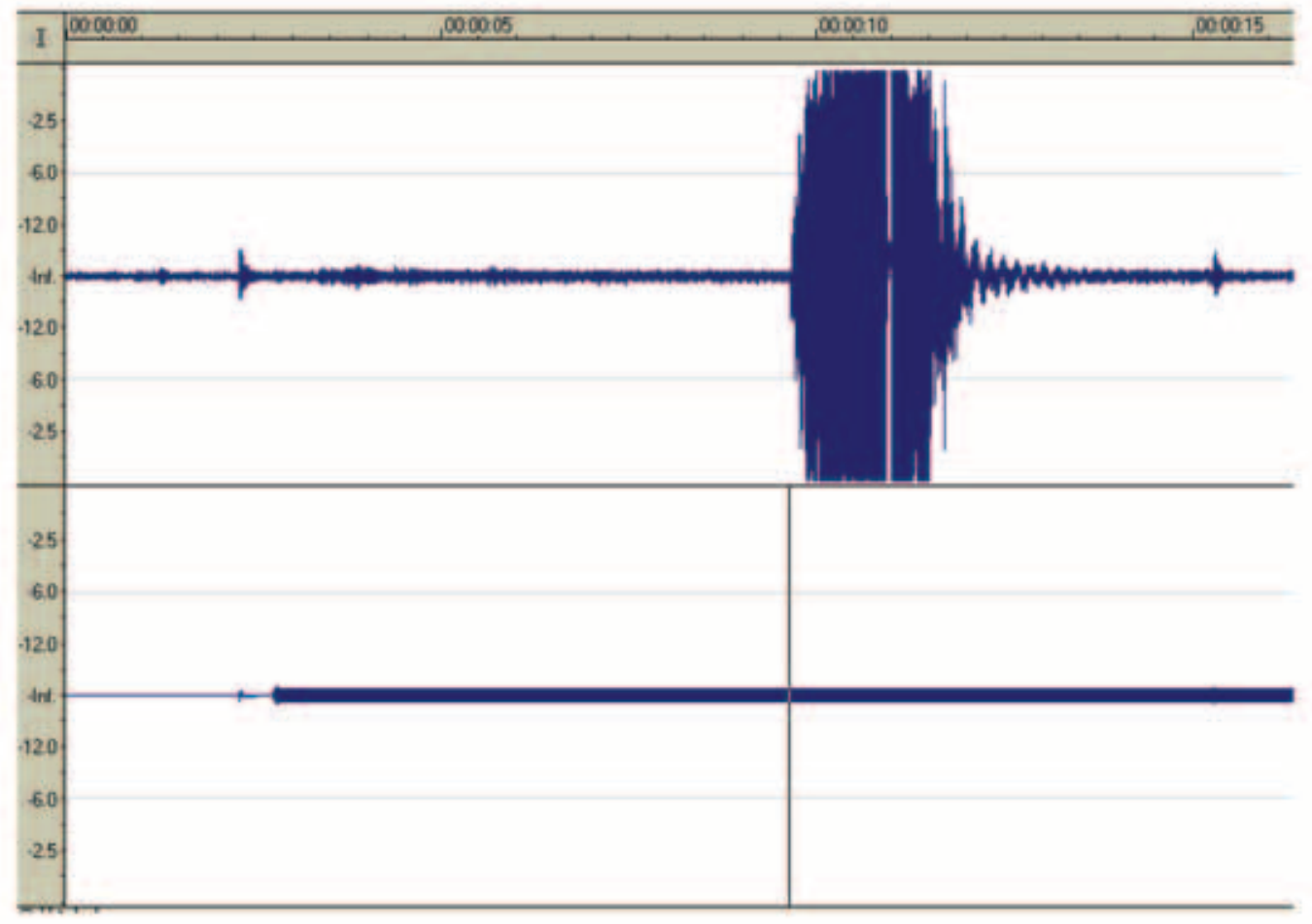

(b)

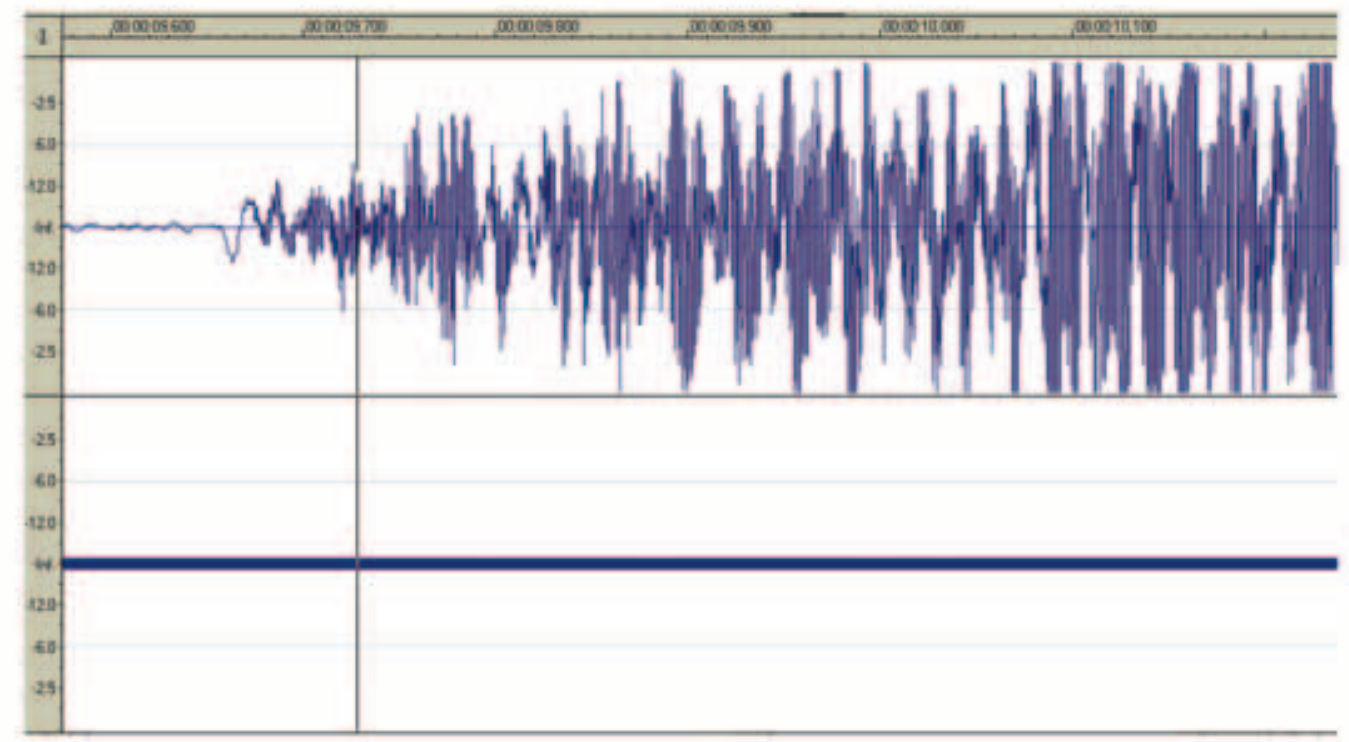

Fig. 7a,b. EM-slip of the labradorite sample; slip happens in the active phase. a) The whole recording; b) the initial part of recording stretched. 
the negative pole. The distribution of EFI proved to be rather mosaic: the values of $\mathrm{V}$ along the perimeter of slip surface vary from 0.01 to $57 \mathrm{~V}$ and the average value is around $8 \mathrm{~V}$ for the separation of probe sites of the order of $1 \mathrm{~mm}$. These values are in quantitative accordance with above theoretical calculations.

We can conclude that electrical impact of the order of several volts per millimeter can induce slip in the system close to the critical state.

\subsection{Assessment of mechanical equivalent of electrical impact}

For assessment of mechanical equivalent of electrical impact, both direct and theoretical methods were used.

In the first case, the mechanical force, initiating slip at the same angle $\alpha<\alpha_{c}$ set in experiments with EM-impact, was measured by spring (accuracy $\pm 0.01 \mathrm{~N}$ ) and torsion (accuracy $\pm 0.005 \mathrm{~N}$ ) dynamometers. Both methods give comparable results. The force, equivalent to slipinitiating EM-impact is of the order of $0.2 \mathrm{~N}$. Another way to obtain the mechanical equivalent is to calculate it from the general equation of balance of forces for a sample placed on the inclined plane

$$
F=m g(\mu \cos \alpha-\sin \alpha) .
$$

As far as $\mu$ is known $(\mu=0.47)$, slip-initiating force can be calculated for any angle. For example, if $\alpha_{c}$ equals $25^{\circ}$, at $\alpha=24^{\circ} 50^{\prime}$ the initiating force is $0.42 \mathrm{~N}$. This value is of the same order as in direct experiments $(0.2 \mathrm{~N}=2$ $10^{4}$ dyne).

\subsection{Statistics of EM-slips}

It is well known that surface phenomena are rather complicated and influenced by many factors: surface roughness, configuration of asperities, humidity, temperature, chemistry of contacting materials, etc.

So it is quite natural that, in the case of contact of rough surfaces, only a small part of the EMpulses initiate motion, namely the pulses that are applied at the most favourable arrangement of asperities on the slip surface.

A general idea on the efficiency of impact can be given by the results of one series of experiment on the basalt sample in which 94 pulses in 30 tests (each test contained several pulses) were applied to the system and 7 of them were accompanied by slip. The total probability of EM-initiation $P$ is thus $7 / 94=0.07$; here the probability of slip during the pulse is 0.04 and that of slip during gap between pulses is 0.03 .

The probabilities of initiation for various stages of impact for this series are: during first pulse - no case; after first pulse -0.01 ; during second pulse -0.02 ; after second pulse -0.01 ; during third pulse -0.02 , etc. Of course, these probabilities vary from one series to another one but the total probability does not change considerably.

It should be noted that the observed process is extremely complicated and its mechanism is not clear. Nevertheless, the experiments show that EM-initiation of slip in mechanical system that is close to the critical state is quite real.

\subsection{Probable physical mechanism (dry conditions)}

In order to understand the physics of EMslip, it is necessary to consider fundamentals of surface phenomena. Intermolecular and intersurface forces, responsible for adhesion and friction, can be loosely divided into three categories: i) purely electrostatic, arising from the Coulomb interaction between charges; ii) polarization forces arising from the dipole moments, induced by internal (bound charges, dipoles) or external electric field; iii) quantummechanical forces, responsible for covalent bonding and steric interactions. All these forces can act simultaneously, resulting in some total adhesion (friction) force. For friction we have

$$
F_{f}=\mu F_{n}
$$

where $\mu$ is friction coefficient and $F_{n}$ is the normal component of force acting on the body (gravity, compression).

From the above classification, it can be deduced that in principle the external electrical field 
can affect the intersurface adhesion (friction) forces, changing either $\mu$ or $F_{n}$ and thus initiating slip of the body, placed on the inclined plane. Of course, EM-impact can also affect $F_{n}$ of the body containing piezoelectric materials. As far as the EM-activation is clearly observed on samples that are practically free of piezoelectric minerals (basalt), we exclude the piezoelectric effect as a principal mechanism of EM-slip.

Having in mind that quite often initiation of slip was observed after switching off the power, it seems reasonable to accept the hypothesis that the applied EM-pulse affects the electrostatic and/or polarization component of the intersurface adhesive forces (forces due to polarization are acting even in passive periods).

\section{Conclusions}

A phenomenological analysis of the possible physical mechanisms of MHD-induced microseismicity has been done.

An experimental set up was built for laboratory modeling of the impact of electromagnetic (EM) pulses on the stability of the mechanical system, which is close to the critical state. The slope of the supporting plane is considered as an analogue of the tectonic stresses for natural faults.

The preliminary experiments, carried out in dry environment, show that a strong EM-pulse $(1200 \mathrm{~V})$ may induce sliding of a sample of rock (granite, basalt, labradorite) placed on the supporting sample, which is inclined at the slope, close to, but less than the critical angle. Of course, the EM field in our experiments was larger than in the field conditions; nevertheless the experimental proof of possibility of EM-initiation of mechanical instability seems to be important.

The mechanical equivalent of electrical force, initiating slip, was evaluated both theoretically and experimentally: it was of order of $0.20 \mathrm{~N}$ for the slopes of support used in experiment.

The statistics of EM-slips were studied: the probability of EM-activation by series of pulses was found to be in the order of 0.07 .

\section{REFERENCES}

De ArChangelis, L, S. ReDner and A. Coniglio (1986): Multiscaling approach in random resistor and random superconducting networks, Phys. Rev., 34, 4656-4673.

BAK, P., C. TANG and K. WIESENFELD (1988): Self-organized criticality, Phys. Rev., A38, 364-374

Benguigi, L. (1988): Simulation of dielectric failure by means of resistor-diode random lattices, Phys. Rev, B 38, 7211-7214.

VorobyeV, A., M. SAMOKHVALOV and R. IBRAGIMOV (1975): Search of effects due to existence of high local electric fields in the lithosphere, in Seismology of Uzbekistan, Tashkent, 213-221 (in Russian).

KHARITONOV, E. (1983): Dielectric Materials with Heterogeneous Structure, Moscow, Radio and Communication (in Russian), pp. 127.

KING, G., R. STEIN and J. LIN (1994): Static stress changes and the triggering of earthquakes, Bull. Seismol. Soc. Am., 84, 935-953.

Nesvetailov, G. and E. SEREBRIAKOV (1966): Theory and Practice of Electrohydraulic Effect, Minsk (in Russian).

ScHOLZ, C. (1990): The Mechanics of Earthquakes and Faulting (Cambridge Univ. Press, Cambridge), pp. 439.

SIBSON, R. (1994): Crustal stress, faulting and fluid flow, in Geofluids: Origin, Migration and Evolution of Fluids in Sedimentary Basins, edited by J. PARNELL, The Geological Soc. London, 69-84.

Sobolev, G., A. Ponomarev, A. Avagimov and V. ZEIGARNIK (2000): Initiating acoustic emission with electric actions, Report on ESC Conference.

Tarasov, N., H. Tarasova, A. Avagimov and V. ZEIGARNIK (1999): The effect of high-power electromagnetic pulses on the seismicity of Central Asia and Kazakhstan, Volcanology and Seismology (Moscow), 4-5, 152-160 (in Russian). (received February 18, 2002;
accepted October 8, 2002) 